Check for updates

Cite this: RSC Adv., 2019, 9, 12325

Received 27th February 2019

Accepted 15th April 2019

DOI: $10.1039 / c 9 r a 01480 b$

rsc.li/rsc-advances

\section{Layered photochromic films stacked from spiropyran-modified montmorillonite nanosheets $\uparrow$}

\begin{abstract}
Mengke Gan, $\$^{\mathrm{a}}$ Tianliang Xiao, $\$^{\mathrm{a}}$ Zhaoyue Liu (D)*a and Yao Wang*b
Two-dimensional (2D) nanosheets are a class of fascinating host material that demonstrates a high specific surface area for the immobilization of functional molecules. Herein, we describe a layered photochromic film using montmorillonite 2D nanosheets immobilized with spiropyran units, which demonstrates a remarkable and reversible photochromic behavior. The synthesis of the layered photochromic film includes the intercalation and exfoliation of montmorillonite powders into 2D nanosheets using a spiropyran-modified surfactant and a subsequent vacuum filtration. The photochromic units of spiropyran-modified quaternary ammonium groups are immobilized on the surface of montmorillonite 2D nanosheets through an electrostatic interaction after exchanging with the native cations in montmorillonite during the intercalation and exfoliation. The photoisomerization of the spiropyran units between closed-ring spiropyran and open-ring merocyanine upon visible/UV irradiation contributes to the photochromic behavior of the layered film. The color contrast between the coloration and decoloration states of photochromic film is optimized by increasing the amount of spiropyran-modified cationic surfactant during the intercalation and exfoliation process. Our layered films with a visual photochromic behavior may promote their applications for optical data storage, optical switching and chemical sensing.
\end{abstract}

\section{Introduction}

Photochromic molecules which can undergo reversible color change upon the external stimulus of light have attracted much attention for potential applications in optical data storage, optical switching and chemical sensing. ${ }^{1-6}$ Spiropyran is one of the most studied photochromic molecules because of its fast and reproducible color switching resulting from the photoisomerization between the colorless (light yellow) closed-ring spiropyran (SP) form and deeply colored open-ring merocyanine (MC) form..$^{7-10}$ Photochromic films are strongly desired for further applications, and are generally derived from the dispersion of the spiropyran molecules into a polymer matrix or the polymerisation of spiropyran-based monomers. ${ }^{711-13}$ The development of an alternative strategy towards novel photochromic films is essentially important.

${ }^{a}$ Key Laboratory of Bio-Inspired Smart Interfacial Science and Technology of Ministry of Education, School of Chemistry, Beihang University, Beijing 100191, P. R. China. E-mail: liuzy@buaa.edu.cn

${ }^{b}$ Guangdong Provincial Key Laboratory of Optical Information Materials and Technology, Institute of Electronic Paper Displays, South China Academy of Advanced Optoelectronics, South China Normal University, Guangzhou 510006, P. R. China.E-mail:wangyao@m.scnu.edu.cn

$\dagger$ Electronic supplementary information (ESI) available: Photochromism of SP-6 aqueous solution; layered montmorillonite nanosheets; ${ }^{1} \mathrm{H}$ NMR and mass spectrum of SP-6. See DOI: 10.1039/c9ra01480b

$\ddagger$ Mengke Gan and Tianliang Xiao contributed equally to this work.
Two-dimensional (2D) nanosheets are an emerging class of nanomaterials that demonstrate an ultrahigh specific surface area because of their large lateral size and ultrathin thickness. ${ }^{14-19}$ These unique surface characteristics make $2 \mathrm{D}$ nanosheets act as fascinating nanosized building blocks to immobilize functional molecules. Furthermore, the solutionbased processability of $2 \mathrm{D}$ nanosheets is favorable for the fabrication of high-quality layered thin film by a simple method such as vacuum filtration. ${ }^{14,20,21}$ Montmorillonite is one of extensively studied layered clay minerals. The layer spacing of Al-O octahedral sheet sandwiched by two Si-O tetrahedral sheets can be intercalated with functional molecules by exchanging their native cations with organic cations..$^{22-25}$ Several examples have reported that the adsorption of cationic spiropyrans into montmorillonite interlayers through ion exchange for photochromic application..$^{9,26-31}$ However, only a few works focused on the fabrication of photochromic films using spiropyrans-intercalated montmorillonite. Saso et al. fabricated ultrathin spiropyran-montmorillonite hybrid film by the Langmuir-Blodgett method. ${ }^{26}$ Kinashi et al. prepared photochromic films by casting a suspension containing cationic spiropyran-intercalated montmorillonites on glass substrate. ${ }^{28}$ In spite of these works, the development of a stable, uniform and large-area photochromic films with a visual color switch derived from spiropyran-intercalated montmorillonite still remains a challenge. 
Herein, we reported a novel photochromic layered film using montmorillonite 2D nanosheets immobilized with spiropyran units. Two kinds of surfactants containing quaternary ammonium cations were used to intercalate and exfoliate montmorillonite powders into high-quality $2 \mathrm{D}$ nanosheets. The spiropyran-modified quaternary ammonium cations were immobilized on the surface of 2D nanosheets during the intercalation and exfoliation, which acted as the photochromic units. The exfoliated montmorillonite nanosheets were subsequently stacked into a stable photochromic layered film by a vacuum filtration, which demonstrated a remarkable and reversible photochromic behavior.

\section{Results and discussion}

\subsection{Photochromic properties of synthesized spiropyran- modified cationic surfactants}

The solid-stated spiropyran-modified cationic surfactant, $1^{\prime}(6-$ triethylammoniohexyl)-3', $3^{\prime}$-dimethyl-6-nitrospiro(2H-1-benzopyran-2,2'-indoline)bromide (SP-6) was in closed-ring SP state (Fig. 1A). When SP-6 was dissolved into water, an open-ring merocyanine (MC) state was formed spontaneously through the cleavage of the $\mathrm{C}_{\text {spiro }}-\mathrm{O}$ bond by the high polarity of water. ${ }^{7,32}$ The photochromic properties of SP-6 in water were then investigated. As shown in Fig. 1B, the aqueous solution of SP-6 (1 mM) exhibited a pink color because of the spontaneous formation of MC form. The maximal absorption peak in visible region was observed at $503 \mathrm{~nm}$ due to the extended $\pi$-conjugation between the indoline and the benzopyran moieties in MC state (Fig. 1C). ${ }^{7}$ The irradiation of visible light changed the color of solution to be light yellow because of the photoisomerization of MC to SP state. Correspondingly, the absorption peak at $503 \mathrm{~nm}$ almost disappeared. When the SP solution was irradiated by UV light, the characteristic absorption peak at $503 \mathrm{~nm}$ increased gradually following the irradiation time (Fig. S1 $\uparrow$ ). Subsequently, the color of the solution recovered to

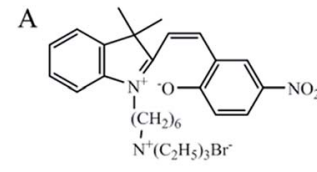

$\mathrm{MC}$

B

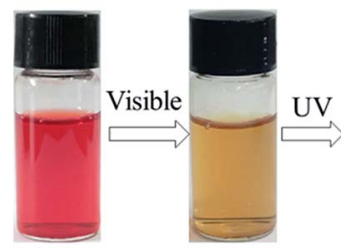

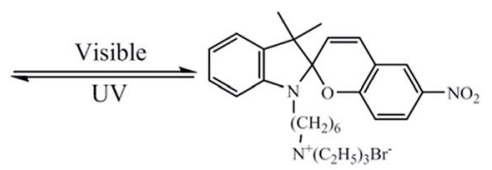

SP
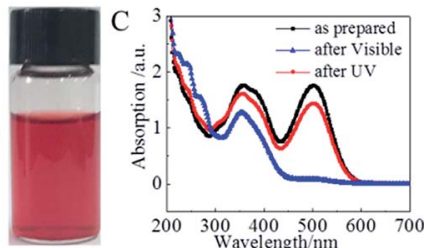

Fig. 1 (A) The photoisomerization between deeply colored merocyanine (MC) and light yellow spiropyran (SP) state in the aqueous solution of a synthesized spiropyran-modified cationic surfactant (SP6). (B) The visual photochromism of SP-6 aqueous solution under visible and the followed UV illumination. (C) The UV-visible absorption spectra for the as-prepared SP-6 solution, the SP-6 solution after visible illumination and the followed UV illumination. be pink color. These results indicated that the aqueous solution of our synthesized spiropyran-modified surfactant demonstrated a clear photochromic property.

\subsection{Characterization of montmorillonite 2D nanosheets immobilized with spiropyran}

It has been well-recognized that montmorillonite powders can be intercalated and exfoliated into $2 \mathrm{D}$ nanosheets through the exchange of its native cations with organic cations in the surfactants, ${ }^{33,34}$ or through the thorough stirring in water. ${ }^{35}$ In our work, two kinds of surfactants were used to intercalate and exfoliate montmorillonite powders. One surfactant is a synthesized SP-6 cationic surfactant. The other one is a commercially available dioctadecyldimethylammonium bromide (DODAB). The quaternary ammonium cations having spiropyran groups in SP-6 acted as the photochromic units. The double octadecyl chains in DODAB were used to improve the dispersion of exfoliated 2D nanosheets in chloroform and enhance the mechanical stability of the photochromic film. As shown in Fig. 2, when montmorillonite powders were stirred with SP-6 and DODAB in water at $70{ }^{\circ} \mathrm{C}$, the quaternary ammonium cations in the surfactants of SP-6 and DODAB exchanged with native cations of $\mathrm{Na}^{+}, \mathrm{Ca}^{2+}$ and $\mathrm{K}^{+}$of montmorillonite. ${ }^{24,25}$ The electrostatic interaction between the quaternary ammonium cations and the negatively charged surface of montmorillonite resulted in the immobilization of spiropyran groups in SP-6 and the double octadecyl chains in DODAB on the surface of montmorillonite 2D nanosheets (Fig. 2). The hydrophobic double octadecyl chains made the dispersion of exfoliated 2D nanosheets in chloroform to form a stable colloidal suspension, which demonstrated a similar photochromism from light pink to light

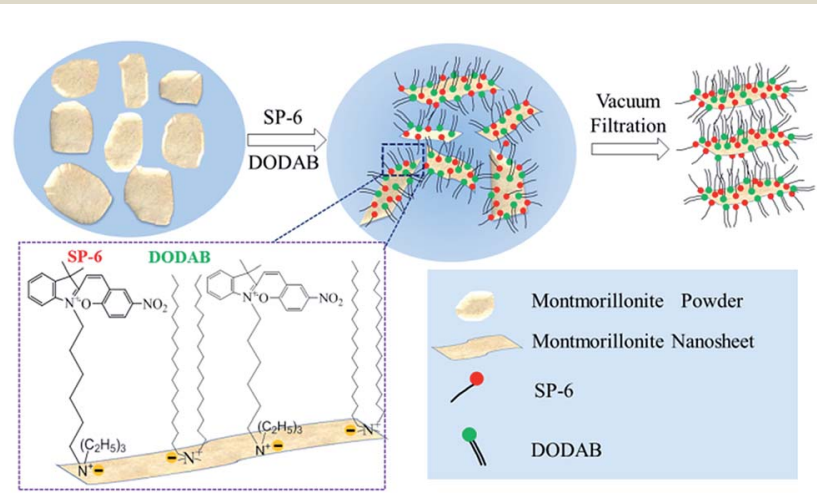

Fig. 2 A schematic diagram for the intercalation and exfoliation of montmorillonite powders into nanosheets using our synthesized SP-6 and commercially available dioctadecyldimethyl ammonium bromide (DODAB) surfactants. The photochromic units of spiropyran-modified quaternary ammonium groups are immobilized on the surface of montmorillonite 2D nanosheets through an electrostatic interaction after exchanging with the native cations in montmorillonite, which could be stacked into a photochromic film by a subsequent vacuum filtration. The double octadecyl chains in DODAB were used to improve the dispersion of exfoliated 2D nanosheets in chloroform and enhance the mechanical stability of the photochromic film. This diagram was not drawn to scale. 
yellow resulting from the photoisomerization between $\mathrm{MC}$ and SP state (Fig. 3A).

The visible irradiation resulted in the decoloration of $2 \mathrm{D}$ nanosheets dispersion solution and the UV illumination recovered its color. In a subsequent process, the stable dispersion of 2D nanosheets immobilized with SP-6 in chloroform was used to fabricate a photochromic film by a vacuum filtration through a nylon filter.

The structure of montmorillonite $2 \mathrm{D}$ nanosheets exfoliated by SP- 6 and DODAB was characterized by TEM measurement. The TEM image (Fig. 3B) indicated that the exfoliated montmorillonite showed a clear 2D nanosheet structure with a lateral size of several hundred of nanometers, which could be used as the building blocks for the formation of a layered film. The AFM measurement also revealed the structure of nanosheets with a similar lateral dimension (Fig. 3C). The height profile indicated that the thickness of montmorillonite 2D nanosheets was $\sim 3.6 \mathrm{~nm}$, which was much larger than that in native montmorillonite $^{34}$ due to the immobilization of quaternary ammonium ions containing spiropyran groups and the double octadecyl chains. ${ }^{36}$ Supposed that the thickness of a pure single-layer montmorillonite nanosheet was $\sim 0.96 \mathrm{~nm},{ }^{37}$ the thickness of quaternary ammonium ions on the two sides of $2 \mathrm{D}$ nanosheets was determined to be $\sim 2.64 \mathrm{~nm}$.

\subsection{Photochromic behaviors of layered films}

The montmorillonite 2D nanosheets in chloroform could be stacked into dark-pink, paper-like layered film by a vacuum filtration of the suspension solution through a nylon filter membrane (inset in Fig. 4A). The surfactant of DODAB was essentially necessary for the intercalation and exfoliation of montmorillonite powers into $2 \mathrm{D}$ nanosheets because the hydrophobic double octadecyl chains could improve the

A
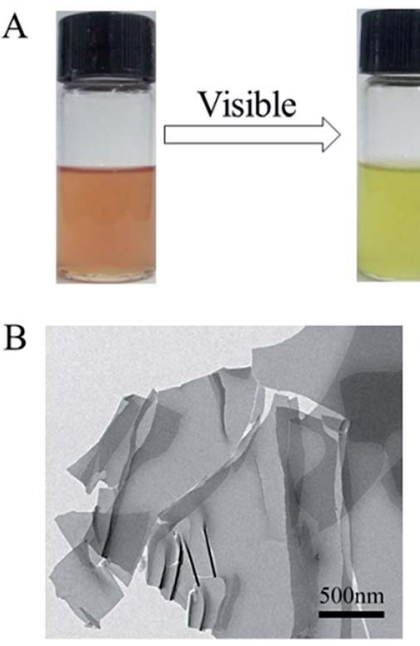

Fig. 3 (A) The montmorillonite 2D nanosheets immobilized with SP-6 in chloroform formed a stable colloidal suspension, which demonstrated a photochromism between light pink and light yellow. (B) The TEM image indicated that the exfoliated montmorillonite showed a 2D nanosheet structure. (C) The AFM measurement revealed the thickness of exfoliated montmorillonite nanosheets was $\sim 3.6 \mathrm{~nm}$.
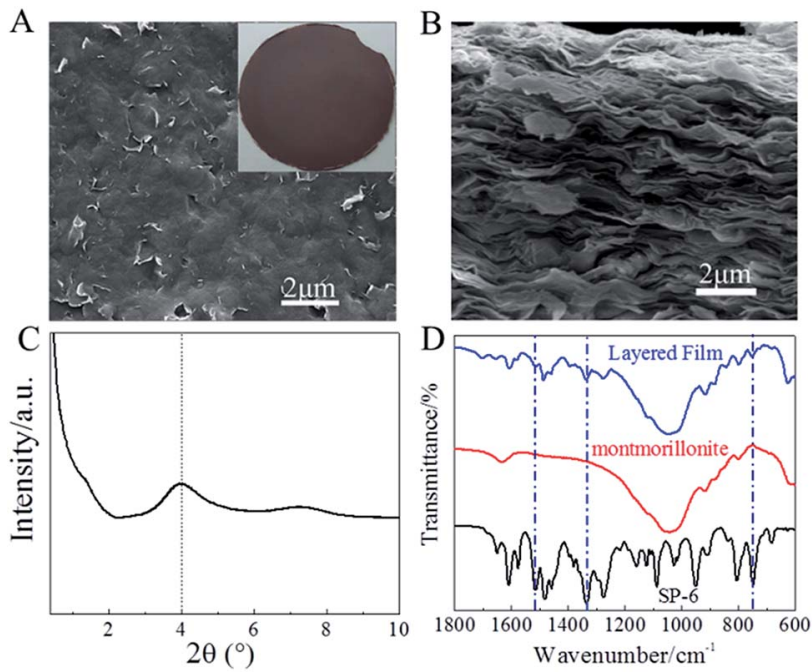

Fig. 4 (A and B) The top-viewed (A) and cross-sectional (B) SEM images of the layered photochromic film prepared by a vacuum filtration of montmorillonite 2D nanosheets immobilized with SP-6. Inset of (A): a photograph of the dark-pink, paper-like and selfstanding layered photochromic film. The dense stacking of montmorillonite nanosheets immobilized with SP- 6 resulted in an oriented layered structure. (C) XRD diffraction pattern of layered photochromic film, which indicated that the calculated $d_{001}$ spacing was $\sim 2.2 \mathrm{~nm}$. (D) The FTIR spectra of SP-6, pure montmorillonite and layered photochromic film. The typical characteristic peaks for the nitro and phenyl groups in SP- 6 were identified in the layered film.

dispersion of exfoliated 2D nanosheets in chloroform. Otherwise, a high-quality layered film could not be obtained. Note that the layered film derived from only DODAB-exfoliated montmorillonite showed a color of light yellow, which was the native color of montmorillonite nanosheets (Fig. S2 $\dagger$ ). The selfstanding layered film demonstrated a smooth surface without obvious defects (Fig. 4A). The wrinkles on the surface indicated the layered film was composed of nanosheets structure. The cross-sectional SEM image (Fig. 4B) verified the dense stacking of nanosheets into an oriented layered structure. The calculated $d_{001}$ spacing in layered film was $\sim 2.2 \mathrm{~nm}$ determined from the $\mathrm{XRD}$ diffraction peak at $2 \theta=4.0^{\circ}$ based on the Bragg's equation $(n \lambda=2 d \sin \theta)$ (Fig. 4C). Note that the very weak XRD diffraction peak at $2 \theta=7.2^{\circ}$ was derived from the small amount of unexfoliated montmorillonite. ${ }^{34}$ The bimolecular layer with a length of $\sim 5.0 \mathrm{~nm}$ formed by DODAB molecules increased the $d_{001}$ spacing of layered film. ${ }^{33}$ The Fourier transform infrared (FTIR) spectra were used to further clarify the existence of spiropyran units in the layered film. In comparison with pure montmorillonite (Fig. 4D), the FTIR spectrum of layered film displayed the typical characteristic peaks for the stretching vibration of nitro group in SP-6 at $1515 \mathrm{~cm}^{-1}$ and $1335 \mathrm{~cm}^{-1}$, and for the bending vibration of $-\mathrm{CH}$ in phenyl groups of SP-6 at $747 \mathrm{~cm}^{-1}, 32$ indicating the successful immobilization of spiropyran units in the layered film.

The self-standing layered film demonstrated a remarkable photochromism at room temperature and ambient pressure. In this case, the weight percentage of SP- 6 in the total surfactants 
during the intercalation and exfoliation process of montmorillonite was $30 \%$. Note that not all the SP-6 molecules were intercalated into montmorillonite. As shown in Fig. 5A-E, the as-prepared self-standing layered film demonstrated a color of dark purple with a strong absorption in visible region due to the MC state of SP-6 (Fig. 5F). The MC state was formed during the intercalation and exfoliation process of montmorillonite by DODAB and SP-6 due to the high polarity of water. The red shift of the characteristic peak of MC from $503 \mathrm{~nm}$ in aqueous solution to $550 \mathrm{~nm}$ in stacked layered film was ascribed to the aggregation of MC in the interlayer space of montmorillonite 2D nanosheets. Following the visible irradiation, the isomerization of MC to SP reduced the visible absorption of the layered film, which decolorized the film to be dark yellow. The UV irradiation was able to recover the color of decolorized film to be dark purple following the recovery of the characteristic peak of $\mathrm{MC}$ at $550 \mathrm{~nm}$. Furthermore, the photochromism of layered film was reversible, as evidenced by the four cycles of coloration and decoloration states (Fig. 5A-E).

\subsection{Enhancement of the visual photochromic contrast of layered films}

Generally, a high spiropyran quantity in the film is strongly desired to enhance the photochromic performance. The quantity of spiropyran in our layered film could be facilely increased by increasing the weight percentage of SP-6 during the intercalation and exfoliation of montmorillonite into $2 \mathrm{D}$ nanosheets. As shown in Fig. 6A and B, when the weight percentage of SP-6 in the total surfactants during the intercalation and exfoliation process increased from $30 \%$ to $70 \%$ and $90 \%$, the color the as-prepared layered film became deeper because of the increased spiropyran quantity in the layered film, which was evidenced by the reduced visible reflectance of $\mathrm{MC}$ in the diffuse reflection spectra (Fig. 6C). Note that the layered film prepared

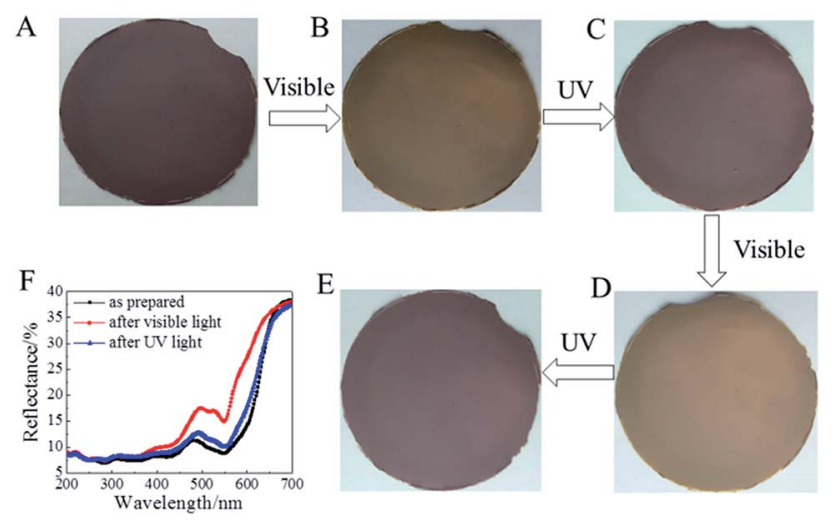

Fig. 5 (A-E) The photochromic behavior of the self-standing layered film. Under visible illumination, the layered membrane demonstrated a photochromism from dark purple to dark yellow (A and B). The UV irradiation recovered the color of decolorized film to be dark purple ( $B$ and $C$ ). The four cycles of coloration and decoloration states (A-E) indicated that the photochromism of layered film was reversible. ( $F$ ) The UV-visible diffuse reflection spectra of the as-prepared layered film and the layered film after visible (decoloration state) and the followed UV illumination (coloration state).
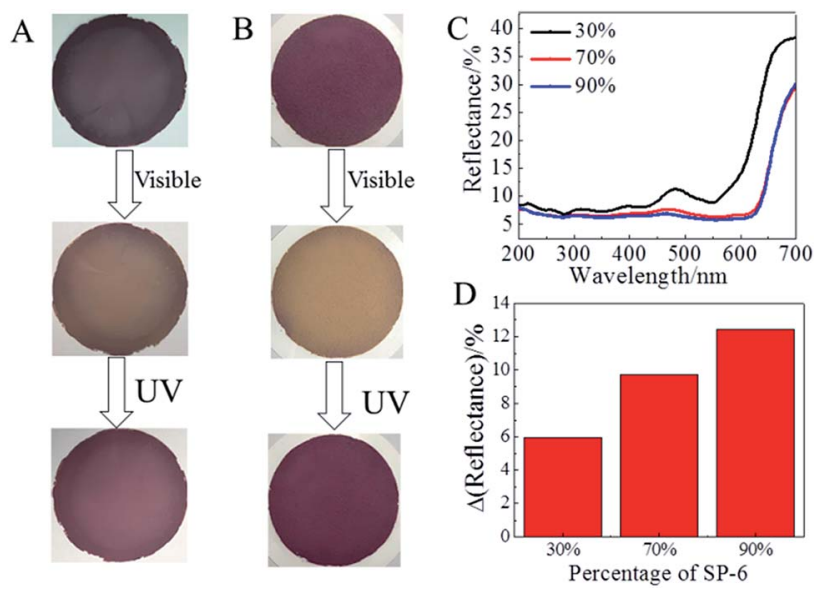

Fig. 6 (A and B) The photochromic behavior of the as-prepared layered film fabricated with different SP-6 quantity. The weight percentage of SP- 6 in the total surfactants was $70 \%$ (A) and $90 \%$ (B). A high spiropyran quantity magnified the visual color contrast between the coloration and decoloration states of the layered film. (C) The UVvisible diffuse reflection spectra of as-prepared layered films when the weight percentage of SP-6 in the total surfactants was 30\%,70\% and $90 \%$. (D) The difference of reflectance at $550 \mathrm{~nm}$ between the coloration and decoloration states of layered films when the weight percentage of SP-6 in the total surfactants was $30 \%, 70 \%$ and $90 \%$.

with $90 \%$ SP-6 was difficult to be peeled off from the nylon filter. More importantly, the high spiropyran quantity magnified the visual color contrast between the coloration and decoloration states of the layered film as shown in Fig. 6A and B. Following the increase of SP- 6 weight percentage in the total surfactants from $30 \%$ to $70 \%$ and $90 \%$ during the intercalation and exfoliation process, the difference between the reflectance of coloration and decoloration states at $550 \mathrm{~nm}$ increased from $5.9 \%$ to $10 \%$ and $12.4 \%$. This magnified visual photochromic contrast might promote the potential application of photochromic films.

\section{Experimental}

\subsection{Synthesis of spiropyran-modified cationic surfactant}

The spiropyran-modified cationic surfactant, 1'(6-triethylammoniohexyl)-3',3'-dimethyl-6-nitrospiro(2H-1-benzopyran2,2'-indoline)bromide ( $\mathrm{SP}-6, \mathrm{C}_{30} \mathrm{H}_{42} \mathrm{~N}_{3} \mathrm{O}_{3} \mathrm{Br}$ ), was synthesized according to the ref. 32 and 38 . The chemical structure was shown in Fig. 1A. The final product was characterized by ${ }^{1} \mathrm{H}$ NMR and mass spectroscopy (Fig. S3 and S4 $\dagger) .{ }^{1} \mathrm{H}$ NMR $\left(\mathrm{CDCl}_{3}\right)$ $\delta: 1.10\left(3 \mathrm{H}, \mathrm{s}, \mathrm{NCCCH}_{3}\right), 1.19\left(3 \mathrm{H}, \mathrm{s}, \mathrm{NCCCH}_{3}\right), 1.28(13 \mathrm{H}, \mathrm{t}$, $\mathrm{N}^{+}\left(\mathrm{CH}_{2} \underline{\mathrm{CH}}_{3}\right)_{3}$ and $\left.\mathrm{N}^{+}\left(\mathrm{CH}_{2}\right)_{2} \underline{\mathrm{CH}}_{2} \underline{\mathrm{CH}}_{2}\right), 1.57-1.64(2 \mathrm{H}, \mathrm{m}$, $\left.\mathrm{NCH}_{2} \underline{\mathrm{CH}}_{2}\right), 1.76\left(2 \mathrm{H}, \mathrm{s}, \mathrm{N}^{+} \mathrm{CH}_{2} \underline{\mathrm{CH}}_{2}\right), 3.04-3.13\left(2 \mathrm{H}, \mathrm{m}, \mathrm{NCH}_{2}\right)$, 3.18-3.24 (2H, m, N $\left.\underline{\mathrm{CH}}_{2}\right), 3.36-3.43\left(6 \mathrm{H}, \mathrm{m}, \mathrm{N}^{+}\left(\underline{\mathrm{CH}}_{2}\right)_{3}\right), 5.81$ (1H, d, H3), 6.48 (1H, d, H4' $), 6.64(1 \mathrm{H}, \mathrm{d}, \mathrm{H} 8), 6.77\left(1 \mathrm{H}, \mathrm{t}, \mathrm{H} 6^{\prime}\right)$, $6.91(1 \mathrm{H}, \mathrm{d}, \mathrm{H} 4), 6.99$ (1H, d, H7 $), 7.09$ (1H, t, H5' $), 7.88-7.93$ $(2 \mathrm{H}, \mathrm{m}, \mathrm{H} 5, \mathrm{H} 7)$ ppm. Mass spectrum: $m / z: 492.3\left(\mathrm{C}_{30} \mathrm{H}_{42} \mathrm{~N}_{3} \mathrm{O}_{3}{ }^{+}\right)$. 


\subsection{Synthesis of the photochromic layered film}

The montmorillonite nanosheets were prepared by the intercalation and exfoliation of montmorillonites powders with an ion exchange capacity of 120 mequiv./100 g (Nanocor, USA, specific surface area: $15-20 \mathrm{~m}^{2} \mathrm{~g}^{-1}$ ) using our synthesized SP-6 and commercially available dioctadecyldimethylammonium bromide (DODAB, Aldrich) in water. ${ }^{33,34}$ The quaternary ammonium cations having spiropyran groups in SP-6 acted as the photochromic units. The double octadecyl chains in DODAB were used to improve the dispersion of exfoliated $2 \mathrm{D}$ nanosheets in chloroform and enhance the mechanical stability of the photochromic film. Typically, $0.2 \mathrm{~g}$ of montmorillonite powders, $51 \mathrm{mg}$ of SP- 6 and $119 \mathrm{mg}$ of DODAB were stirred in $10 \mathrm{~mL}$ of water at $70{ }^{\circ} \mathrm{C}$ for $1 \mathrm{~h}$. The weight percentage of SP- 6 in the total surfactants during the intercalation and exfoliation process of montmorillonite was $30 \%$. After cooling to room temperature, the suspension was centrifuged at $2000 \mathrm{rpm}$. The obtained precipitate was washed with 1:1 chloroform/ methanol, followed by being dispersed into chloroform to form a suspension. The photochromic layered film was obtained by a vacuum filtration of the suspension solution through a nylon filter membrane $(50 \mathrm{~mm}$ diameter and $0.45 \mu \mathrm{m}$ pore size, Shanghai Xinya Company, China). Without specific explanation, a self-standing photochromic film was obtained by peeling off it from the nylon filter after vacuum filtration.

\subsection{Characterization}

${ }^{1} \mathrm{H}$ NMR was recorded on an Agilent $400 \mathrm{MHz}$ NMR spectrometer. The mass spectrum was obtained with an Agilent-1100 LC/ MSD mass spectrometer. The morphology of exfoliated montmorillonite 2D nanosheets was observed by a FEI JEM-1200EX transmission electron microscope (TEM). The thickness of 2D nanosheets was measured using Bruker Dimension Icon atomic force microscope (AFM). The surface and cross-sectional morphology of the stacked layered films were observed using a FEI Quanta FEG 250 environmental scanning electron microscope (SEM). An Ultima IV X-ray diffraction meter (Rigaku Corporation, Japan) was used to determine the interlayer spacing of layered film. The UV-visible absorption and diffuse reflection spectra were recorded using a UV-3600 spectrometer (Shimadzu, Japan). The Fourier transform infrared (FTIR) spectra were measured on a Nicolet 6700 spectrometer (Thermo).

\subsection{Photochromic characterization}

The photochromism of the SP-6 aqueous solution and the layered film from the photoisomerization between the deeply colored merocyanine (MC) and light yellow spiropyran (SP) states was carried out by irradiation of visible light with an irradiance of $100 \mathrm{~mW} \mathrm{~cm}^{-2}$ from a Xe lamp (Beijing Perfectlight Technology Co. Ltd, China). The recovery to MC form was achieved by irradiation of $365 \mathrm{~nm}$ ultraviolet (UV) light with an irradiance of $10 \mathrm{~mW} \mathrm{~cm}{ }^{-2}$ from a super-high pressure mercury lamp (CHF-XM500, Beijing Trusttech Co. Ltd, China). The corresponding absorption changes were characterized by the UV-visible absorption and diffuse reflection spectra.

\section{Conclusions}

In summary, we have successfully fabricated a novel layered photochromic film using montmorillonite 2D nanosheets immobilized with spiropyran units. The nanosheets were prepared by the intercalation and exfoliation of montmorillonite using a synthesized spiropyran-modified cationic surfactant and a commercial cationic surfactant with double octadecyl chains in water. The spiropyran-modified quaternary ammonium groups were immobilized on the surface of $2 \mathrm{D}$ nanosheets by the cationic exchange during the intercalation and exfoliation, which acted as the photochromic units. The $2 \mathrm{D}$ nanosheets were stacked into a photochromic layered film by a vacuum filtration, which demonstrated a remarkable and reversible photochromic behavior.

\section{Conflicts of interest}

There are no conflicts to declare.

\section{Acknowledgements}

This work was supported by National Key Research and development Program of China (2017YFA0206902, 2017YFA0206900), Beijing Municipal Science \& Technology Commission (No. Z181100004418013), National Natural Science Foundation of China (21571011 and 51673007), the Fundamental Research Funds for the Central Universities (YWF-19-BJ$\mathrm{J}$-115) and the Startup Foundation from SCNU (No. 8S0134).

\section{Notes and references}

1 M. Zhu, L. Zhu, J. J. Han, W. Wu, J. K. Hurst and A. D. Q. Li, J. Am. Chem. Soc., 2006, 128, 4303-4309.

2 S. Kawata and Y. Kawata, Chem. Rev., 2000, 100, 1777-1788.

3 H. Tian and S. Yang, Chem. Soc. Rev., 2004, 33, 85-97.

4 J. Zhang, Q. Zou and H. Tian, Adv. Mater., 2013, 25, 378-399.

5 M. Irie, Chem. Rev., 2000, 100, 1685-1716.

6 S. Coiai, E. Passaglia, A. Pucci and G. Ruggeri, Materials, 2015, 8, 3377-3427.

7 R. Klajn, Chem. Soc. Rev., 2014, 43, 148-184.

8 Y. Funasako, A. Takaki, M. Inokuchi and T. Mochida, Chem. Lett., 2016, 45, 1397-1399.

9 K. Takagi, T. Kurematsu and Y. Sawaki, J. Chem. Soc., Perkin Trans. 2, 1991, 1517-1522.

10 T. Yamaguchi and M. Ogawa, Chem. Lett., 2018, 47, 189-191. 11 K. Uchida, A. Takata, M. Saito, A. Murakami, S. Nakamura and M. Irie, Adv. Mater., 2003, 15, 785-788.

12 M. Levitus and P. F. Aramendía, J. Phys. Chem. B, 1999, 103, 1864-1870.

13 G. Berkovic, V. Krongauz and V. Weiss, Chem. Rev., 2000, 100, 1741-1753. 
14 C. Tan, X. Cao, X. Wu, Q. He, J. Yang, X. Zhang, J. Chen, W. Zhao, S. Han, G. Nam, M. Sindoro and H. Zhang, Chem. Rev., 2017, 117, 6225-6331.

15 Y. Chen, Z. Fan, Z. Zhang, W. Niu, C. Li, N. Yang, B. Chen and H. Zhang, Chem. Rev., 2018, 118, 6409-6455.

16 C. Tan, Z. Lai and H. Zhang, Adv. Mater., 2017, 29, 1701392.

17 H. Jin, C. Guo, X. Liu, J. Liu, A. Vasileff, Y. Jiao, Y. Zheng and S. Qiao, Chem. Rev., 2018, 118, 6337-6408.

18 J. Wan, S. D. Lacey, J. Dai, W. Bao, M. S. Fuhrer and L. Hu, Chem. Soc. Rev., 2016, 45, 6742-6765.

19 D. Chimene, D. L. Alge and A. K. Gaharwar, Adv. Mater., 2015, 27, 7261-7284.

20 J. Kang, V. K. Sangwan, J. D. Wood and M. C. Hersam, Acc. Chem. Res., 2017, 50, 943-951.

21 K. Shehzad, Y. Xu, C. Gao and X. Duan, Chem. Soc. Rev., 2016, 45, 5541-5588.

22 B. N. Narayanan, R. Koodathil, T. Gangadharan, Z. Yaakob, F. K. Saidu and S. Chandralayam, Mater. Sci. Eng., B, 2010, 168, 242-244.

23 J. Shi, H. Liu, Z. Lou, Y. Zhang, Y. Meng, Q. Zeng and M. Yang, Comput. Mater. Sci., 2013, 69, 95-99.

24 Y. Xi, R. L. Frost and H. He, J. Colloid Interface Sci., 2007, 305, 150-158.

25 W. Xie, Z. Gao, W. Pan, D. Hunter, A. Singh and R. Vaia, Chem. Mater., 2001, 13, 2979-2990.
26 N. Saso, T. Yamamoto, Y. Umemura and Y. Einaga, Colloids Surf., A, 2008, 317, 309-315.

27 K. Takagi, T. Kurematsu and Y. Sawaki, J. Chem. Soc., Perkin Trans. 2, 1995, 1667-1671.

28 K. Kinashi, H. Kita, M. Misaki, Y. Koshiba, K. Ishida, Y. Ueda and M. Ishihara, Thin Solid Films, 2009, 518, 651-655.

29 T. Seki and K. Ichimura, Macromolecules, 1990, 23, 31-35.

30 H. Tomioka and T. ltoh, J. Chem. Soc., Chem. Commun., 1991, 532-5533.

31 H. Nishikiori, R. Sasai, K. Takagi and T. Fujii, Langmuir, 2006, 22, 3376-3380.

32 C. Sun, K. Arimitsu, K. Abe, T. Ohkubo, T. Yamashita, H. Sakai and M. Abe, Mater. Technol., 2004, 22, 229-237.

33 Y. Okahata and A. Shimizu, Langmuir, 1989, 5, 954-959.

34 T. Xiao, Q. Liu, Q. Zhang, Z. Liu and J. Zhai, J. Phys. Chem. C, 2017, 121, 18954-18961.

35 H. Yao, Z. Tan, H. Fang and S. Yu, Angew. Chem., Int. Ed., 2010, 49, 10127-10131.

36 H. He, Y. Ma, J. Zhu, P. Yuan and Y. Qing, Appl. Clay Sci., 2010, 48, 67-72.

37 H. Sun, J. Zhang, L. Li, J. Xu and D. Sun, Colloids Surf., A, 2013, 426, 26-32.

38 H. Sakai, H. Ebana, K. Sakai, K. Tsuchiya, T. Ohkubo and M. Abe, J. Colloid Interface Sci., 2007, 316, 1027-1030. 